\title{
Zero-Point Oscillations in the Planck Vacuum State and Its Coordinate Uncertainty
}

\author{
William C. Daywitt
}

\begin{abstract}
This short paper argues that the charged quantum oscillators in the quasi-continuum Planck vacuum (PV) state are responsible for the zero-point oscillations in that state. The Planck particle (PP) quantum energy levels for the oscillators are derived from first principles. The PV coordinate uncertainty concerning the PV structure easily follows from these results.
\end{abstract}

Index Terms-PP Oscillator, Zero-Point Oscillations, PV Coordinate Uncertainty.

\section{INTRODUCTION}

$\mathbf{T}$ HE Planck vacuum theory supports a 7-dimensional spacetime that consists of one observed and one unobserved 4-dimensional spacetime. The following development calculates the PP zero-point oscillator fields associated with the 4-dimensional observed spacetime, and reveals the intimate connection between these oscillators and the uncertainty of the underlying PV space. That space consists of a continuum that is pervaded by a degenerate collection of PP cores-it is the discrete nature of this collection that leads to the PV coordinate uncertainty.

The theoretical foundation [1] [2] [3] [4] of the PV theory rests upon the unification of the Einstein, Newton, and Coulomb superforces:

$$
\frac{c^{4}}{G}\left(=\frac{m_{*} c^{2}}{r_{*}}\right)=\frac{m_{*}^{2} G}{r_{*}^{2}}=\frac{e_{*}^{2}}{r_{*}^{2}} \rightarrow r_{*} m_{*} c=\frac{e_{*}^{2}}{c} \quad(=\hbar)
$$

where the ratio $c^{4} / G$ is the curvature superforce that appears in the Einstein field equations. $G$ is Newton's gravitational constant, $c$ is the speed of light, $m_{*}$ and $r_{*}$ are the Planck mass and length respectively [5, p.1234], and $e_{*}$ is the massless bare (or coupling) charge. The fine structure constant is given by the ratio $\alpha \equiv e^{2} / e_{*}^{2}$, where $e$ is the observed electronic charge magnitude. The ratio $e_{*}^{2} / c$ to the right of the arrow is the spin coefficient for the PP, the proton, and the electron cores, where $\hbar$ is the reduced Planck constant.

The $e_{*}^{2}$ in (1) is the squared coupling charge, where one of the $e_{*} \mathrm{~s}$ belongs to the PP core $\left( \pm e_{*}, m_{*}\right)$ under consideration, and the other charge belongs to any one of the remaining PP cores making up the degenerate PV state. The product $e_{*}^{2}=$ $\left( \pm e_{*}\right)^{2}$ implies that both the PP and its antiparticle contribute to the PV oscillator density. The antiparticle is found in the unobserved 4-dimensional spacetime.

DOI: http://dx.doi.org/10.24018/ejers.2021.6.4.2438

Published on May 13, 2021.

William C. Daywitt is retired from the National Institute of Standards and Technology, Boulder, CO 80305 USA (e-mail: wcdaywitt@me.com).
Equating the Einstein and Coulomb superforces from (1) leads to the force

$$
F_{*}\left(r_{*}\right)=\frac{e_{*}^{2}}{r_{*}^{2}}-\frac{m_{*} c^{2}}{r_{*}}=0 \quad \text { with } \quad r_{*}=\frac{e_{*}^{2} / c}{m_{*} c}
$$

the separate PP cores $\left( \pm e_{*}, m_{*}\right)$ exert on the degenerate PV state. This equation can be used to model the PP oscillator.

Section II derives the basic quantum oscillator energy (16) by quantizing the Planck action associated with the corresponding classical, nonrelativistic energy equation. Section III discusses coordinate uncertainty, and comments and conclusions are found in Section IV.

\section{Planck Particle Oscillator}

The vanishing of the force in (2) can be used to derive the harmonic oscillator equations for the PP core. For small $x / r_{*}$ with the core $\left( \pm e_{*}, m_{*}\right)$ at $r=r_{*}$ :

$$
\begin{gathered}
F_{*}\left(x+r_{*}\right)=\frac{e_{*}^{2}}{\left(x+r_{*}\right)^{2}}-\frac{m_{*} c^{2}}{x+r_{*}}=\frac{e_{*}^{2} / r_{*}^{2}}{\left(1+x / r_{*}\right)^{2}}-\frac{m_{*} c^{2} / r_{*}}{1+x / r_{*}} \\
\approx-2 \frac{e_{*}^{2}}{r_{*}^{2}} \frac{x}{r_{*}}+\frac{m_{*} c^{2}}{r_{*}} \frac{x}{r_{*}}=-\frac{e_{*}^{2}}{r_{*}^{2}} \frac{x}{r_{*}}=-\frac{m_{*} c^{2}}{r_{*}} \frac{x}{r_{*}}
\end{gathered}
$$

$(\mathbf{x}=x \hat{\mathbf{r}})$ where the equality to the right of the arrow in (1) is used in the calculation.

Equation (3) yields the oscillator force

$$
\begin{gathered}
-K_{*} x=-\frac{e_{*}^{2}}{r_{*}^{2}} \frac{x}{r_{*}}=-\frac{m_{*} c^{2}}{r_{*}} \frac{x}{r_{*}} \\
=-\frac{e_{*}^{2}}{r_{*}^{3}} x=-\frac{m_{*} c^{2}}{r_{*}^{2}} x
\end{gathered}
$$

that defines the "spring constant" $K_{*}$, and leads to the harmonic oscillator equation

$$
m_{*} \ddot{x}=-K_{*} x=-\frac{m_{*} c^{2}}{r_{*}^{2}} x
$$

or

$$
\ddot{x}=-\frac{K_{*}}{m_{*}} x=-\omega_{*}^{2} x
$$

where

$$
\omega_{*}=\left(\frac{K_{*}}{m_{*}}\right)^{1 / 2}=\frac{c}{r_{*}}=\frac{1}{t_{*}}=\frac{m_{*} c^{2}}{e_{*}^{2} / c}
$$

and $t_{*}\left(=r_{*} / c\right)$ is the Planck time [5, p.1233]. The final ratio on the right represents the PP mass energy divided by its spin coefficient $e_{*}^{2} / c$.

The solution to (6) $[6, \mathrm{p} .208]$ used here is

$$
x=x_{0} \sin \omega_{*} t
$$




$$
\dot{x}=\omega_{*} x_{0} \cos \omega_{*} t .
$$

The oscillator energy corresponding to (3)-(9) is

$$
\begin{gathered}
u_{*}=\frac{m_{*} \dot{x}^{2}}{2}+\frac{K_{*} x^{2}}{2}=\frac{m_{*} \omega_{*}^{2} x_{0}^{2}}{2}\left(\cos ^{2} \omega_{*} t+\sin ^{2} \omega_{*} t\right) \\
=\frac{m_{*} \omega_{*}^{2} x_{0}^{2}}{2}=m_{*} c^{2} \frac{x_{0}^{2}}{2 r_{*}^{2}}=\hbar \omega_{*} \frac{x_{0}^{2}}{2 r_{*}^{2}}=A \omega_{*}
\end{gathered}
$$

where

$$
A=\hbar \frac{x_{0}^{2}}{2 r_{*}^{2}}
$$

(The separate PP cores within the degenerate PV state are confined to spheres of diameter no larger than about $2 r_{*}$. Thus the PPs in the PV state are nonrelativistic and the nonrelativistic equation (10) is appropriate.)

The quantization of the Planck action (11) then takes the form

$$
A_{n}=\hbar \frac{x_{n}^{2}}{2 r_{*}^{2}}
$$

for $n=(0,1,2, \cdots)$, leading to the differences

$$
\overbrace{A_{n}-A_{n-1}}^{\hbar}=\hbar\left(\frac{x_{n}^{2}-x_{n-1}^{2}}{2 r_{*}^{2}}\right)
$$

yielding

$$
\left(\frac{x_{n}^{2}-x_{n-1}^{2}}{2 r_{*}^{2}}\right)=1
$$

for the PP coordinate-differentials. If the PV spacetime were a continuum, then $\hbar=0$ and the coordinate-differentials vanish-it is the pervasion of this continuum by the PP cores that leads to the PV coordinate uncertainty and to $\hbar \neq 0$.

Equation (14) can be solved for $x_{n}^{2} / 2 r_{*}^{2}$ in a straightforward iterative manner (Appendix A) and leads to

$$
\frac{x_{n}^{2}}{2 r_{*}^{2}}=\frac{1}{2} \frac{x_{0}^{2}}{r_{*}^{2}}+n=\frac{1}{2}+n
$$

where it is natural to set $x_{0}=r_{*}$, as it is at the Compton radius $r_{*}$ in (3) that the PP oscillations (6) take place. Substituting (15) into (10), and using (1), then yields

$$
\left(u_{*}\right)_{n}=\left(\frac{1}{2}+n\right) \hbar \omega_{*}=\left(\frac{1}{2}+n\right) \frac{e_{*}^{2}}{r_{*}}
$$

whose zero-point $(n=0)$ solution is

$$
\left(u_{*}\right)_{0}=\frac{\hbar \omega_{*}}{2}=\frac{e_{*}^{2} / r_{*}}{2} .
$$

\section{COORDINATE UNCERTAINTY}

Equations (13) and (14)

$$
\overbrace{A_{n}-A_{n-1}}^{\hbar}=\hbar\left(\frac{x_{n}^{2}-x_{n-1}^{2}}{2 r_{*}^{2}}\right)=\hbar
$$

lead to the obvious coordinate-differential uncertainty

$$
\delta A_{n}=\frac{\overbrace{A_{n}-A_{n-1}}^{\hbar}}{2}=\frac{\hbar}{2}\left(\frac{x_{n}^{2}-x_{n-1}^{2}}{2 r_{*}^{2}}\right)=\frac{\hbar}{2} .
$$

\section{COMMENTS AND CONCLUSIONS}

As pointed out in the Introduction, the solution (17) only applies to the 4-dimensional observed spacetime. However, the 4-dimensional unobserved spacetime yields the same solution; so the 7-dimensional spacetime solution (including the PP and its antiparticle) is

$$
2\left(u_{*}\right)_{0}=\hbar \omega_{*}=\frac{e_{*}^{2}}{r_{*}}
$$

where $e_{*}^{2} / r_{*}$ is the cosmic energy potential that permeates the entire PV space. Finding this solution and the coordinate uncertainty is the purpose of the present paper. Except for the one reference (Appendix B), this particular vacuum energy is unrecognized in the open literature.

\section{APPENDIX A}

ITERATIVE SOLUTION

For $n=(0,1,2, \cdots)$, (14) leads to

$$
\begin{gathered}
\frac{x_{n}^{2}-x_{n-1}^{2}}{2 r_{*}^{2}}=1 \\
\frac{x_{n-1}^{2}-x_{n-2}^{2}}{2 r_{*}^{2}}=1 \\
\ldots \ldots \ldots \ldots \ldots \ldots . . . \ldots . . . \\
\frac{x_{2}^{2}-x_{1}^{2}}{2 r_{*}^{2}}=1 \\
\frac{x_{1}^{2}-x_{0}^{2}}{2 r_{*}^{2}}=1 .
\end{gathered}
$$

Summing equations $A_{n}$ through $A_{1}$ then yields

$$
\frac{x_{n}^{2}-x_{0}^{2}}{2 r_{*}^{2}}=n
$$

for the total PP coordinate-differential.

\section{APPENDIX B SPACE Potential}

130:7.6 There are seven different conceptions [dimensions] of space as it is conditioned by time. Space is measured by time, not time by space. The confusion of the scientist grows out of failure to recognize the reality of space. Space is not merely an intellectual concept of the variation in relatedness of universe objects. Space is not empty, and the only thing man knows which can even partially transcend space is mind. Mind can function independently of the concept of the spacerelatedness of material objects. Space is relatively and comparatively finite to all beings of creature status. The nearer consciousness approaches the awareness of seven cosmic dimensions, the more does the concept of potential space approach ultimacy. But the space potential is truly ultimate [fully understood] only on the absolute level. [7, 130:7.6] [4] 


\section{REFERENCES}

[1] P. Davies, Superforce: the Search for a Grand Unified Theory of Nature. Simon and Schuster, Inc., New York, 1984.

[2] W.C. Daywitt, "A Model for Davies' Universal Superforce", Galilean Electrodynamics, Sept./Oct., 83, 2006.

[3] W.C. Daywitt, "The Trouble with the Equations of Modern Fundamental Physics", American Journal of Modern Physics. Special Issue: "Physics without Higgs and without Supersymmetry", v. 5, no. 1-1, 22, 2016. See also www.planckvacuum.com.

[4] W.C. Daywitt, Comparing the Planck-Vacuum and the Urantia-Book Depictions of the Seven-Dimensional Spacetime, European Journal of Engineering Research and Science, Vol.5, No.12, December, 2020.

[5] B.W. Carroll, D.A. Ostlie, An Introduction to Modern Astrophysics, (Addison-Wesley, San Francisco, Boston, New York, Cape Town, Hong Kong, London, Madrid, Mexico City, Montreal, Munich, Paris, Singapore, Sidney, Tokyo, Toronto, 2007)

[6] W.P. Allis, M.A. Herlin, Thermodynamics and Statistical Mechanics, (McGraw-Hill Book Co., New York, Toronto, London, 1952).

[7] Multiple Authors. The Urantia Book. Urantia Foundation, www.urantiaDOTorg, 1955. 